\title{
Performance Analysis of a New Type of Automated Container Terminal
}

\author{
Wei Yan ${ }^{1}$, Yishi Zhu ${ }^{1}$ and Junliang $\mathrm{He}^{1}$ \\ ${ }^{1}$ Engineering Research Center of Container Supply Chain Technology, Ministry of \\ Education, Shanghai Maritime University, Shanghai 201306, P. R. China \\ weiyan@shmtu.edu.cn
}

\begin{abstract}
Container transportation has gradually become the main direction of the deyelopment of shipping today. With the ship's large-scale, specialization and modernization the development of handling capacity of modern container terminal ploys a key role in low-cost transportation, and efficient circulation. Automated contamerterminal can not only improve the utilization rate of equipment, reduce operating costs, but also greatly improves the efficiency of terminal. This paper analyzes the efficient and economical automation container terminal based on the frame-bridge handling teghnology of transport vehicles independently developed by the ZPMC And on this basis put forward the improvement of handling technology, and compare models created to validate the supertority of its improvement plan.
\end{abstract}

Keywords: Automated container terminal, multistory frame bridge, handling technology, performance analysis

\section{Introduction}

With the container shipping volume increased significantly all over the world and the trend of large container ships, how to effectively improve the efficiency of stevedoring vessels, reducing operating costs has become the focus of the industry's workers [1]. In this situation, planning personnel bave also-accepted the idea of automation container terminal design and many domestic and foreign experts and scholars who contribute positively to the automated terminal handling technology research has also focused on it, such as Zhu Minghua [2] analyzed in detail by double 40ft container gantry cranes, low bridges and rail distribution system consisting of a new type of handling technology in automatic container terminal. Analysis of operation characteristics of quayside and low-bridge loading and unloading operations of the new technology system, and calculate the operating efficiency of critical process equipments. Finally, the simulation proved its effectiveness. Liu C I [3] analyzed the worldyide major ports in the application of advanced handling equipment, and analyzed ZPMC independent research for efficient economical automated container terminals, AGVbased and ALV-based automated container terminal handling process by the simulation. Shi Fei, Zhang Xinyan, et al. [4] for the port's future expected amount of work to calculate the automated container terminal handling equipment inside each number, and determine the overall layout of the pier, on the basis of the pier, the unloading processes were simulated, and verify that the average working time of unloading meet the pre-set amount of port operations. Wang Wei, et al. [5] based on the traditional container terminal handling technology, introduce the combination of line-based automated bridges and rail gantry. Automatic carrier (AGV) cooperates with rail gantry crane; DRMG and CRMG based 
handling technology of automated container terminal. Zhaoyan Hu [6] described a new type of structure, characteristics, work process of bridge crane, automated container stacking technology and some several key technical issues. Presents a new automated warehousing technology automated container handling technology. Lu Zhen, et al. [7] analyze two different kinds of automated container terminal, and transport systems were compared and analyzed by establishing evaluation index. Finally, advantages and disadvantages of these two solutions are verified by simulation. Hyo Young Bae [8] the level of transport system based on AGV and ALV are compared and analyzed. By adjusting the mechanical equipment operation rate obtain the compared model of these two transportation system. When the double trolley quayside container cranes are adopted, the efficiency of ALV is much higher than that of AGV.

Based on the previous studies, this paper presents a new type of horizontal thansport system based on multi-layer frame bridge. Wharf Apron mainly utilize the combination of high and low frame bridges, and the yard also adopt multi-layers frame bridge. The transport vehicles can interfere with each other freely on these layers between the shore and yard. And in this transport system, the adjustment of the layout of the yard, to recluce the moving distance of the ARMG, so as to improve operating efficiency of the ARMG. It also can improve the utilization of the yard. Finally, through the case studies, obtain handling efficiency of these two terminals with the different layers of frame bridges, and prove that the new type of handing technology can improve the handling efficiency.

\section{Automated Container Terminal Handling Techmology Analysis}

\subsection{Design and layout}

The new handing technology or the ACT is different from the technology developed by the ZPMC. Its frame bridge is multi-layered, the frame bridge layers coordinate with the number of transport yehicles each or in order to achieve maximum operational efficiency.

A half track layout scheme in each storage yard is not only occupying the yard space resource, but also hindering the travelling of horizontal transport equipment. The multilayer is set up to assure that the loading, unloading and collection operations are processing at the same time, and the transport vehicles can travel with no interference on the frame bridges, effectively avoid Relay operations, reducing the field of bridge carts running distance $\mathrm{t}$ also effectively avoids the relay operation of yard crane, and reduces the running distance of yard bridge crane. Moreover, a plurality of transport channels can be selected from the front to the container yard areas. It effectively reduces the waiting situation, so as to reduce the loading and unloading time. The frame bridge of wharf and yard area are shown in Figure 1:

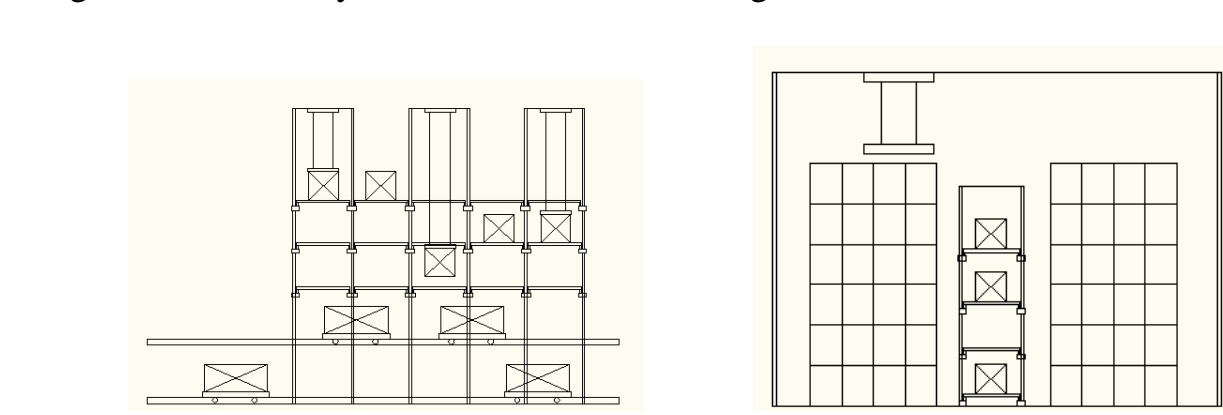

Figure 1. Schematic diagram of the frame bridges 
Yard layout is also improved by utilizing the scheme, which adopt left and right stacking plan and set multistory frame bridge rail intermediately from the quayside to the yard area. Such as the external trucks can complete the collection without entering the yard, it directly put the container on the behind of the container yard, then the vehicles transport to the inside yard. Frame bridges of the yard setting in the middle can reduce the moving distance of the trolley of yard crane in the horizontal direction. A multi-layers frame bridge replacing one and a half of single track improve the space utilization, especially when the longitudinal length of the pier is relatively long. The layout of new type of automated container terminal is shown in Figure 2:

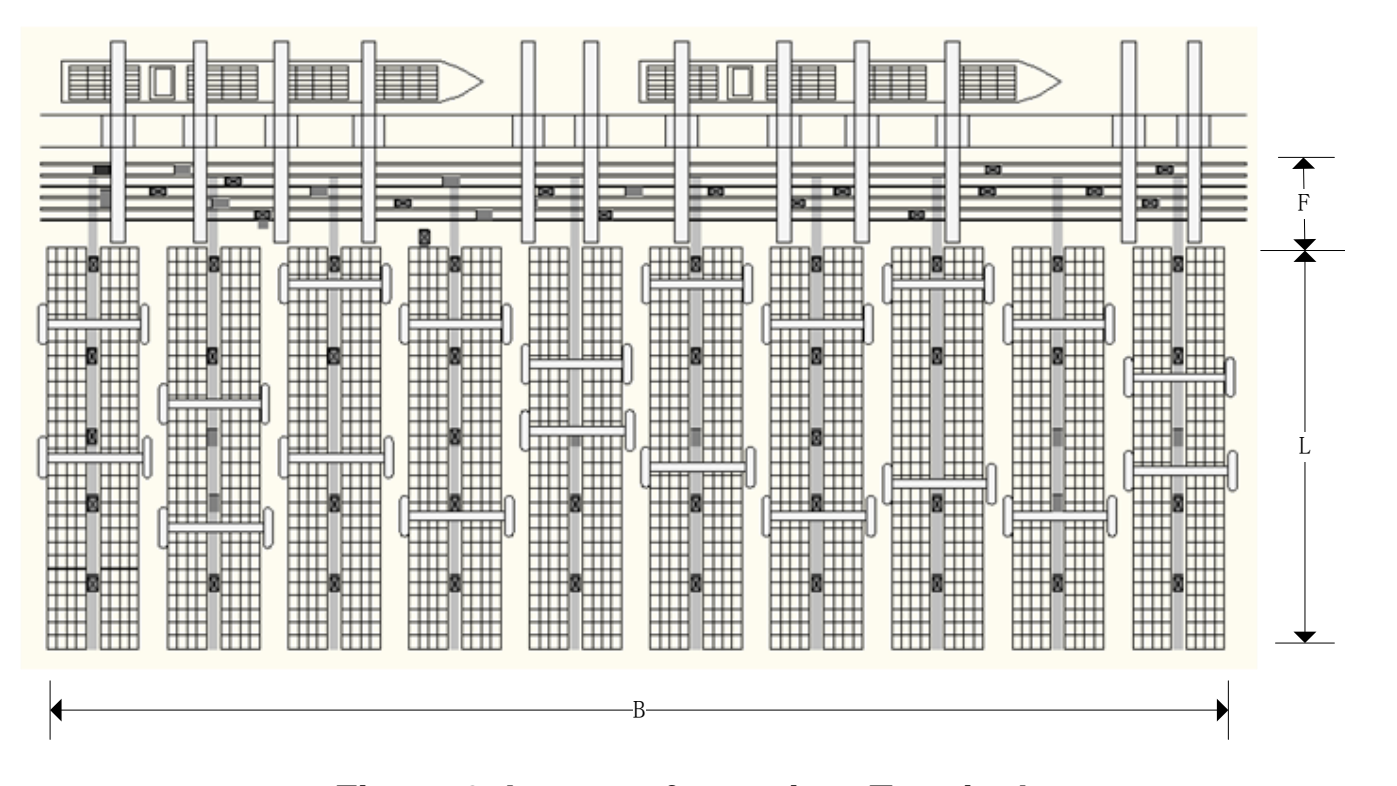

Figure 2. Layout of container Terminal

\subsection{Introduction of handling technology}

The loading and unloading process as an example, container ship berthing firstly, the crane will hoisted containers from the ship and then sent to the crane frame platform while unlocking container lock artificially (it was only manned operation of automated terminal). Then the landside trolley lift the container to the low bridge transport vehicle (LV), the container will be transported to the designated position of transfer container area, the low bridge crane is responsible for shifting the container to the yard transport vehicl $(Y \mathrm{~V}$ ). YV carry the container into the designated slit of yard area. When the yard crane arrives, the container will be transported to the specified position. The loading and unloading process is similar to the inverse process. The flow chart of one loading and unloading cycle is shown in Figure 3. 


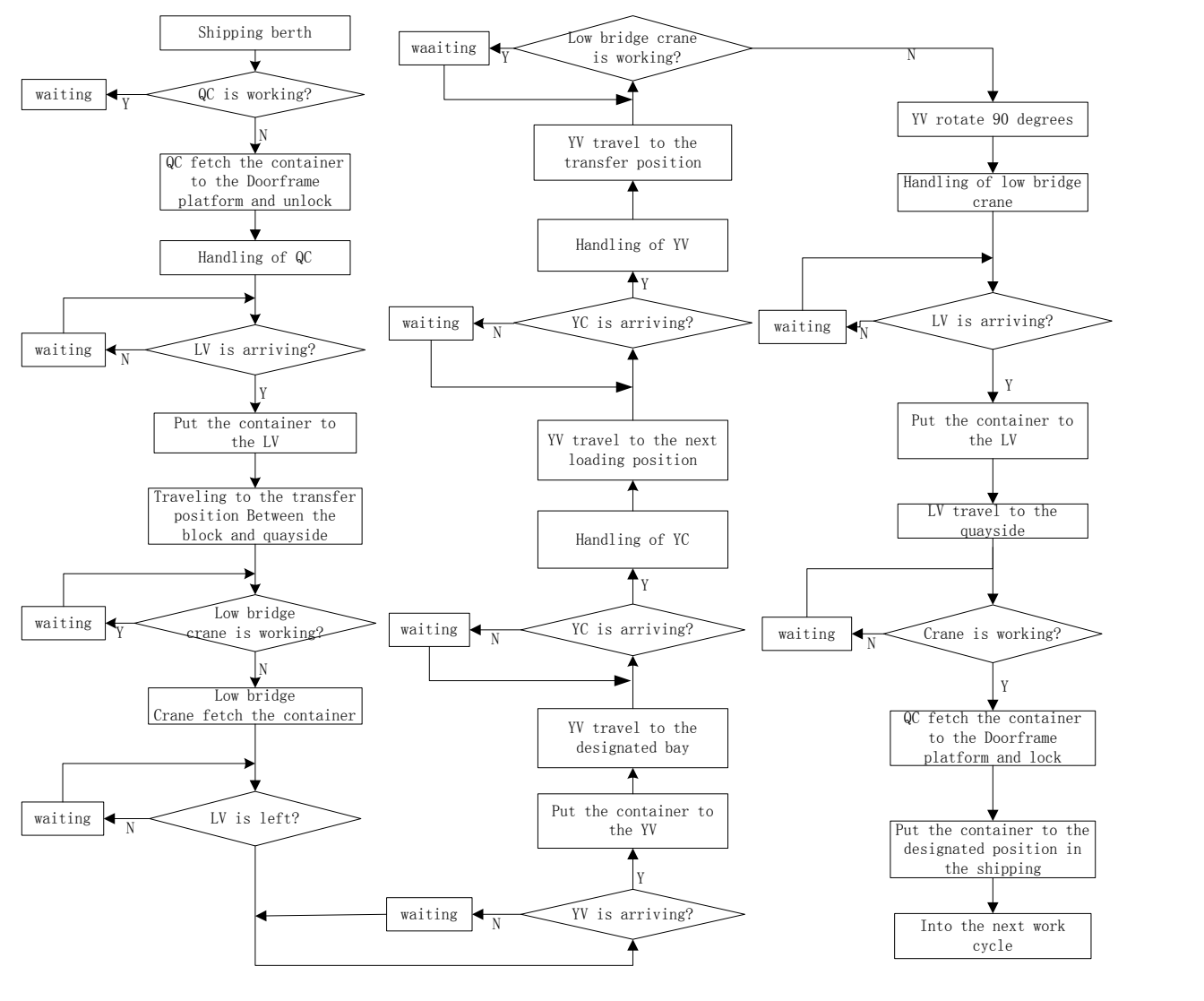

Figure 3. Flowchart ôl loading and unloading

Handling and transportafion equipment of automated container terminal are shown in Table 1. Quay quaysiae adopt contalner crane with dual $40 \mathrm{ft}$ double-trolley, it can also grab two 40-foot containers of eight 20 -foot containers. Horizontal transport equipment along the coastline adopts low bridge cranes and low bridge transport vehicles, and the perpendicular direction use yard transport vehicles. In the back yard, gantry cranes are adopted. Its device parameters as follows:

Table 1. Parameters of equipment

\begin{tabular}{|c|c|c|c|c|c|c|c|}
\hline \multirow[t]{2}{*}{ Type } & \multirow[t]{2}{*}{ Equipment } & \multicolumn{4}{|c|}{ Running speed } & \multicolumn{2}{|c|}{ Acceleration time } \\
\hline & & Load lift & Unload lift & Trolley & Crane & Trolley & Crane \\
\hline Lifting & Dual 40ft QC & $1.25 \mathrm{~m} / \mathrm{s}$ & $30 \mathrm{~m} / \mathrm{s}$ & $4 \mathrm{~m} / \mathrm{s}$ & $0.75 \mathrm{~m} / \mathrm{s}$ & $6 s$ & $6 s$ \\
\hline & YCs & $0.5 \mathrm{~m} / \mathrm{s}$ & $1 \mathrm{~m} / \mathrm{s}$ & $2 \mathrm{~m} / \mathrm{s}$ & $1.67 \mathrm{~m} / \mathrm{s}$ & $3 \mathrm{~s}$ & $6 s$ \\
\hline & Low bridge crane & $0.5 \mathrm{~m} / \mathrm{s}$ & $1 \mathrm{~m} / \mathrm{s}$ & \multicolumn{2}{|c|}{$2 \mathrm{~m} / \mathrm{s}$} & \multicolumn{2}{|c|}{$6 s$} \\
\hline Horizontal & LV & \multicolumn{4}{|c|}{$5 \mathrm{~m} / \mathrm{s}$} & \multicolumn{2}{|c|}{$8 s$} \\
\hline transport vehicle & YV & \multicolumn{4}{|c|}{$5 \mathrm{~m} / \mathrm{s}$} & \multicolumn{2}{|c|}{$8 \mathrm{~s}$} \\
\hline
\end{tabular}

\subsection{Features of Handling Technology}

(1) In the actual operation process, loading, unloading, collection and fetching are simultaneous. Using of multi-story frame bridges, the transport vehicles can shuttle on 
the arbitrary story, which can avoid the waiting problems caused by operation of equipments at the same time.

(2) The utilization rate of yard space is improved obviously. Yard adopt symmetrical layout of each block, and arrange multi-layer frame bridge in the middle of each block. This layout is not only improving the utilization of storage space greatly, but also reducing the moving distance of trolley of the yard crane in the direction parallel to the shoreline.

(3) The external trucks do not enter the yard during collection but directly lift the container to the yard transport vehicle by yard crane behind the container block. So the yard need not set the lanes, not only improves the utilization of yard, reduce cost, but also conducive to the realization of full automatic operation in the yard.

\section{Performance Analyses}

\subsection{Comparison of stockpiling capacity}

Suppose area of two ACT is equal to each other. Yard layout of two kinds of automated container terminal is shown in Figure 4. Span width of YCs is W, vertical length of the block is $L$, the length of the contanner is $L_{T E U}$, width and height of the container are equal is $\mathrm{W}_{\mathrm{TEU}}$. The number of containers is $W / W_{T E U}$ along the shoreline; the number of containers is $L / L_{T E U}$ vertical shoreline. The number of container is $H$ in the height direction. Then the two yard contains the number of containers are:

$$
\begin{aligned}
& C_{F B}=\frac{B}{W} \cdot \frac{L}{L_{T E U}} \cdot\left(\frac{W}{W_{T E U}}-3\right) \cdot H \\
& C_{F B}{ }^{\prime}=\frac{B}{W^{\prime}} \cdot \frac{L}{L_{T E U}} \cdot\left(\frac{W^{\prime}}{W_{T E U}}-2\right) \cdot H
\end{aligned}
$$

Which $C_{F B}$ means that the original proposal storage capacity, $\mathrm{CFB}$ ' means the new type storage capacity.
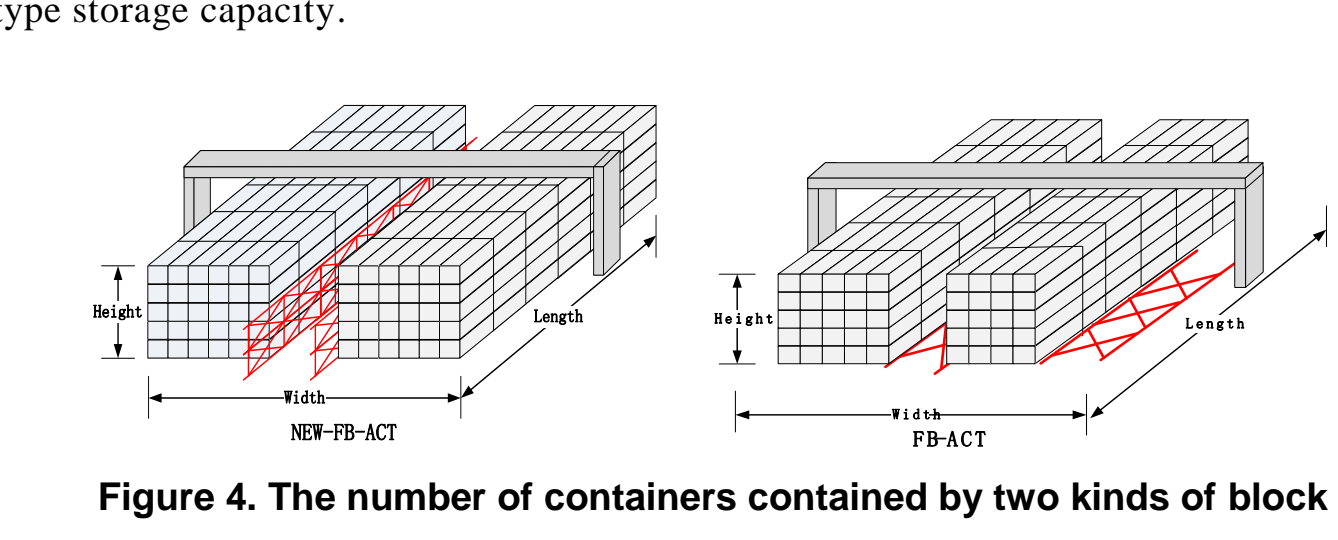

Figure 4. The number of containers contained by two kinds of block

For fair comparison, the number of bay of two ACT is equal, and then the two container storage capacity ratio is $\frac{C_{F B}}{C_{F B^{\prime}}}=\frac{W / W_{T E U}-3}{W / W_{T E U}-2}$. If we take the number of container

in each row is 14 within one block, the annual container throughput of traditional plan is 2 million TEU, the new can accommodate 2.18 million TEU, stockpiling capacity increased by $9 \%$. 


\subsection{Comparison of Horizontal Transport Efficiency}

(1) Establish transport efficiency indicators

In order to complete a container a cyclic process of handling operations, regardless of shipping or unloading operations, must go through this cycle. The cycle time required can be expressed as follows:

$$
T_{c}=h_{Q C}+h_{Y C S}+T_{Y V}+T_{L V}
$$

Which, $h_{Q C}$ represents the handling time of QCs as well as the waiting time of LVs for QC complete the operation. $h_{Y C S}$ represents the handling time of YCs as well as the waiting time of YV for YC complete the operation. $T_{Y V}$ indicates the travelling time of YV to complete a handling cycle, ie $T_{Y V}=2 t_{Y V}+w_{Y V} . T_{L V}$ indicates the travelling time of LV to complete a loading/unloading job cycle, ie $T_{L V}=2 t_{L V}+w_{L V} \cdot w_{Y V}$ and $w_{L V}$ are the waiting ime of $\mathrm{YV}$ and $\mathrm{LV}$ for the TP. The cycle can be split into two parts, one is YV to complete a job cycle and the other is LV to complete a job cycle. These two parts can be expressed as.

$$
\begin{aligned}
& O_{Y V}=2 t_{Y V}+w_{Y V}+h_{Y C} \\
& O_{L V}=2 t_{L V}+w_{L V}+h_{Q C}
\end{aligned}
$$

1) Calculate $t_{Y V}$, which compute A and B distance between two points in Figure 5. As the transport vehicles parked in the designated position beside the frame bridge in each block, it should adopt a discrete variable method togalculate the expected value. $L_{A B}$ is calculated as follows:

$$
\begin{aligned}
& O A=\sum_{i=1}^{r} i \cdot L_{F B} \cdot \frac{1}{r} \\
& O B=\sum_{i=1}^{n} i \cdot L_{T E U} \cdot \frac{1}{n}
\end{aligned}
$$

Where $\mathrm{n}$ is the number of contaners in the vertical direction of shoreline, $n=L / L_{T E U} ; L_{F B}$ is each column width of the br dge framework, and $L_{F B}=F / r ; r$ is the number of columns of the bridge frame, i.e. $d_{Y V}$ and $d_{Y V}$ can be expressed as follows:

$$
d_{Y V}=d_{Y V}^{\prime}=O A+Q B==\sum_{i=1}^{r} i \cdot L_{F B} \cdot \frac{1}{r}+\sum_{i=1}^{n} i \cdot L_{T E U} \cdot \frac{1}{n}
$$

According to the formulation $t_{Y V}=d_{Y V} / v_{Y V}$, we can obtain $t_{Y V}$ and $t^{\prime}{ }_{Y V}$.

2) Calculate $t_{L V}$, assuming the lifting and fetch occurred in the A point. The running distance from an arbitrary point $\mathrm{P}$ to point $\mathrm{A}$ is $d_{L V}, A$ and $P$ are respectively with $\mathrm{N}$ kinds of possible positions, so $(\mathrm{A}, \mathrm{P})$ have $N^{2}$ possibilities. The means distance between two points $\mathrm{A}$, $\mathrm{P}$ is calculated as follows:

$$
d_{L V}=\sum_{i=1}^{N}\left\{\left(\sum_{j=0}^{N-i} j+\sum_{k=1}^{i-1} k\right) \cdot \frac{B}{N} \cdot \frac{1}{N}\right\} \cdot \frac{1}{N}
$$


Similarly, we have $d^{\prime}{ }_{L T}$ :

$d_{L V}{ }^{\prime}=\sum_{i=1}^{2 N}\left\{\left(\sum_{j=0}^{2 N-i} j+\sum_{k=1}^{i-1} k\right) \cdot \frac{B}{2 N} \cdot \frac{1}{2 N}\right\} \cdot \frac{1}{2 N}$

According to the formulation $t_{L V}=d_{L V} / v_{L V}$, we can obtain $t_{L V}$ and. $t_{L V}^{\prime}$

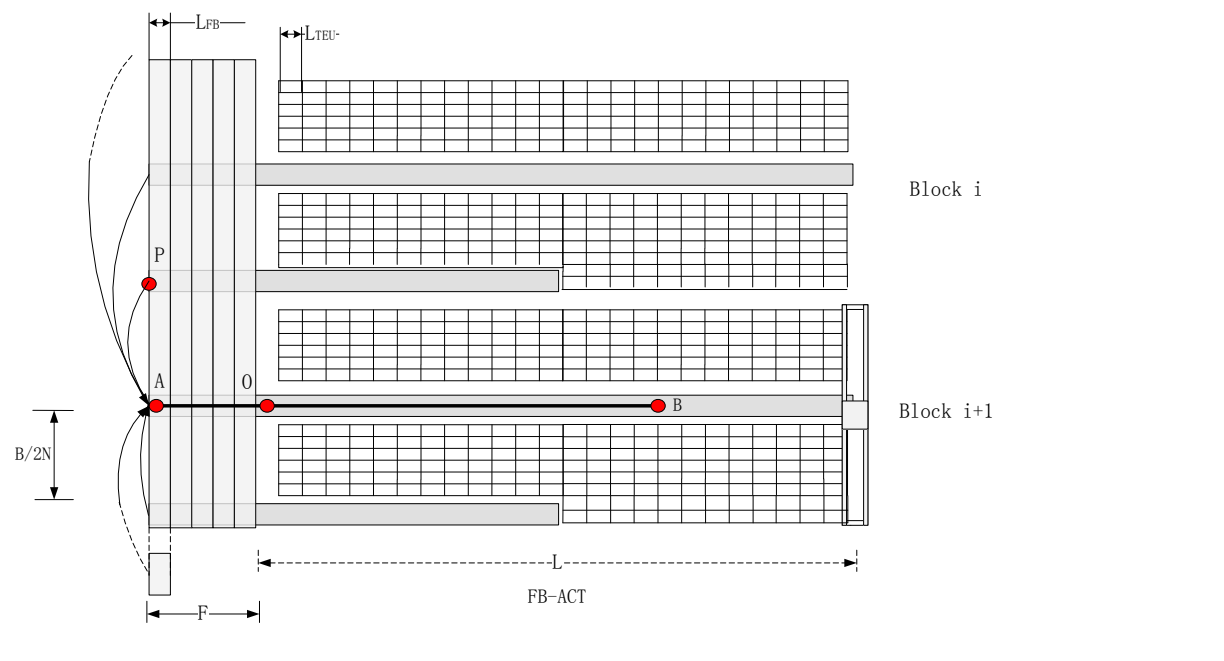

Figure 5. The calculation or $d_{y v}$ and $d_{L V}$

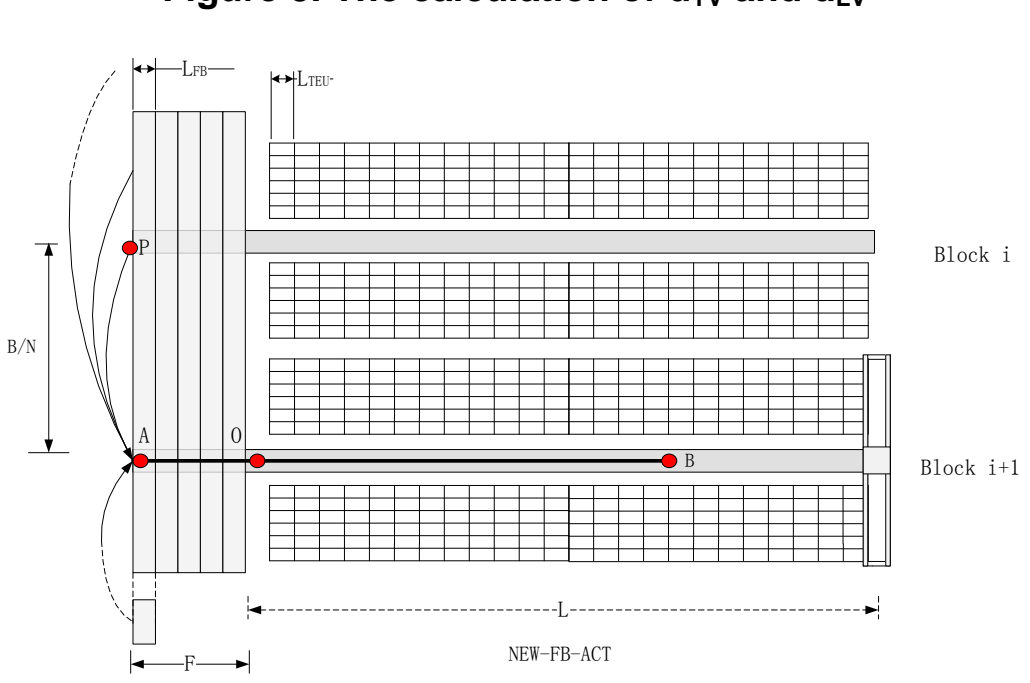

Figure 6. The calculation of $d^{\prime}{ }_{Y V}$ and $d^{\prime}{ }_{L V}$

3) Calculate the waiting time of $w_{L V}$, the running process of LV is simplified as Figure 7. Queuing theory is established to solve the model [9]. This process can be expressed as M/M/S model, the first $M$ mean that the arriving process of LV (Poisson) is Poisson flow, second $M$ represents the LV service process time obey negative exponential distribution, $S$ presents the service system include $\mathrm{S}$ service platform, and, where $s_{Y V}$ is the layer of the rail in the yard. 


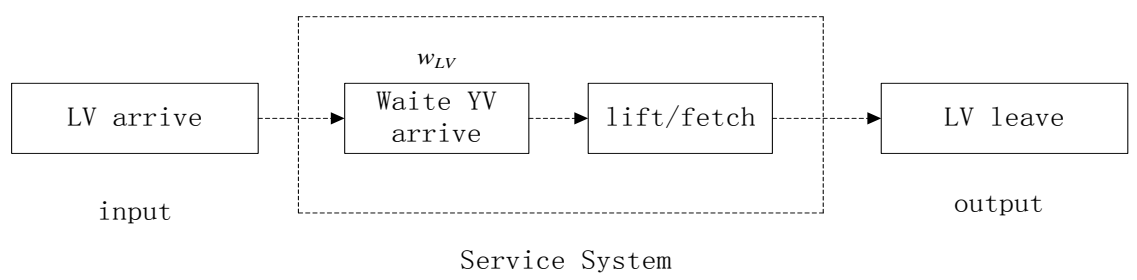

Figure 7. Service processes of LVs

According to mean waiting time formula of $\mathrm{M} / \mathrm{M} / \mathrm{S}$ queuing theory model, we can get the average waiting time $w_{L v}$ :

$$
w_{Y V}=\frac{(S \rho)^{S}}{S !} \cdot\left[(1-\rho) \sum_{n=0}^{S-1} \frac{(S \rho)^{n}}{n !}+\frac{(S \rho)^{S}}{S !}\right]^{-1} \cdot \frac{t}{(1-\rho) \cdot S}
$$

Where, $\rho=(\lambda \cdot t) \cdot S$ indicates the traffic intensity of the yard; $\lambda$ ind cates the average arrival rate of $L V ; t=2 t_{Y V}+h_{Y C S}$ indicates the time from YV leaving TP to coming TP once again.

Assuming in a long period of operation time $T$, the number of job cycle that one LV complete is $T / O_{L V}$. Then the total number of cycles in each column of frame bridges is $M_{L V} \cdot s_{L V} \cdot T / O_{L V}$, at the same time one TP need to complete $M_{L V} \cdot s_{L V} \cdot T /\left(M_{T P} \cdot O_{L V}\right)$ cycles. Wherein, $M_{L V}$ is the number of LV in each column and each story; $s_{L V}$ is the number of umber of stories of frame bridge in quayside The average rate of LV can be got through the above analysis, i.e. the number of LV reach to the TP in a unit of time is shown as:

$$
\lambda=M_{L V} \cdot s_{L V} /\left(M_{T P} \cdot O_{L V}\right)
$$

The traffic intensity pepresents the number of object reaching a service platform within a certain period of time $T$ it can be expressed as:

$$
\rho=\lambda \cdot t / S=\frac{M_{L V} \cdot s_{L V} \cdot t}{M_{T P} \cdot O_{L V} \cdot S} \frac{M_{L V} \cdot s_{L V} \cdot t}{M_{T P} \cdot\left(2 t_{L V}+w_{L V}+h_{Q C}\right) \cdot S}
$$

Combined with formula (12), (13) and equation (4), waiting time of LV, i.e. $w_{L V}$ can be obtained.

4) Calculation $w_{Y V}$, in the long period of time, $M_{Y V} \cdot s_{Y V} \cdot N$ YVs and $M_{L V} \cdot r \cdot s_{L V}$ LVs complete cycles of loading and unloading should be equal. Since the average efficiency of each transport vehicle respectively are $1 / O_{Y V}$ and $1 / O_{L V}$, then $M_{Y V} \cdot s_{Y V} \cdot N / O_{Y V}=M_{L V} \cdot r \cdot s_{L V} /$ $O_{L V}$ B $\mathrm{tth}$ sides of the equation can be expressed that the horizontal transport efficiency of the system 1.e. $M_{Y V} \cdot s_{Y V} \cdot N /\left(2 t_{Y V}+w_{Y V}+h_{Y C}\right)=M_{L V} \cdot r \cdot s_{L V} /\left(2 t_{L V}+w_{L V}+h_{Q C}\right) \cdot w_{Y V}$ can be obtained from his equation.

(2)Comparison of horizontal transport efficiency

Horizontal transport efficiency of the system can be presented as $M_{Y V} \cdot s_{Y V} \cdot N / O_{Y V}$ and $M_{L V} \cdot r \cdot s_{L V} / O_{L V}$, and $O_{L V}=2 t_{L V}+w_{L V}+h_{Q C}$, then $\eta_{F B}^{\prime}$ can be expressed as :

$$
\eta_{F B}=M_{L V} \cdot r \cdot s_{L V} / O_{L V}=M_{L V} \cdot r \cdot s_{L V} /\left(2 t_{L V}+w_{L V}+h_{Q C}\right)
$$


Useing the same method to solve $\eta_{F B}^{\prime}$, replace $s_{Y V}, s_{L V}, M_{Y V}$ and $h_{Y C}$ With $s_{Y V}^{\prime}, s_{L V}^{\prime}, M_{Y V}^{\prime} h_{Y C}^{\prime}$.

\section{Case Study}

Through the efficiency obtained by the above, transport efficiency of two terminals can be compared in different conditions. Before the comparison, we determine the value of each parameter first [9], show as follows:

Table 2. Values of the parameters of two kinds of ACT

\begin{tabular}{|c|c|c|}
\hline Original plan & New plan & Explanation \\
\hline Variable values & Variable values & \\
\hline$N=10$ & $N=10$ & Number of blocks \\
\hline$S_{Y V}=1 ; s_{L V}=1$ & $s_{Y V}^{\prime}=1,2,3,4 ; s_{L V}^{\prime}=1$ & Number of stories of frame bridge \\
\hline$r=5$ & $r=5$ & Number of rows of frame bridge in quay siden \\
\hline$M_{T P}=2$ & $M_{T P}=2$ & Number of TP on each column of frame bridge \\
\hline$M_{Y V}=2$ & $M_{Y V}^{\prime}=2 *_{S}$ & Number of YV in each block \\
\hline$M_{L V}=1$ & $M_{L V}=1,2,3,4$ & Number of LV in each row and story of frame bridge \\
\hline$L_{F B}=5 m$ & $L_{F B}=5 m$ & width Quay frame b \\
\hline$h_{Q C}=1 \min$ & $h_{Q C}=1 \min$ & Handling time of QC of NEW-FB-ACT and FB-ACT \\
\hline$h_{Y C}=\operatorname{lmin}$ & $h_{Y C}^{\prime}=f(s) \min$ & $\begin{array}{l}\text { Handling time of } \mathrm{YC} \text { of NEW-FB-ACT and FB-ACT, } \\
\text { the yalue is related to the number of YV }\end{array}$ \\
\hline
\end{tabular}

According to the setting of parameters in Table 1 and Table 2, it could obtain comparison of transportation efficiency of two kinds of ACT using Matlab when the frame bridge with different layers and different number of LV in each layer of the frame bridge. But because the layers of frame bridges are limited by the height of yard crane and the quay crane, the layer of track in the block adopt up 104 layers. The efficiency ratio values are shown in the Table 3 :

Table 3. Comparison of the horizontal transportation efficiency

\begin{tabular}{|c|c|c|c|c|}
\hline \multicolumn{5}{|c|}{$\eta_{F B}^{\prime} / \eta_{F B}$} \\
\hline \multicolumn{5}{|c|}{$M_{T P}=1$} \\
\hline Layer & $I L=$ & $M_{L V}=2$ & $M_{L V}=3$ & $M_{L V}=4$ \\
\hline$s_{Y V}^{\prime}=2$ & & 1.498 & 1.531 & 1.536 \\
\hline$s_{Y V}^{\prime}=3$ & 1.669 & 2.104 & 2.142 & 2.154 \\
\hline$s_{Y V}^{\prime}=4$ & 1.782 & 2.548 & 2.695 & 2.649 \\
\hline \multicolumn{5}{|c|}{$M_{T P}=2$} \\
\hline Layer & $M_{L V}=1$ & $M_{L V}=2$ & $M_{L V}=3$ & $M_{L V}=4$ \\
\hline & 0.933 & 1.155 & 1.251 & 1.310 \\
\hline$s_{Y V}^{\prime}=3$ & 1.528 & 1.896 & 1.993 & 2.109 \\
\hline$s_{Y V}^{\prime}=4$ & 1.586 & 2.063 & 2.188 & 2.222 \\
\hline
\end{tabular}

In order to analyze the data more intuitive, the line chart is as follows: 

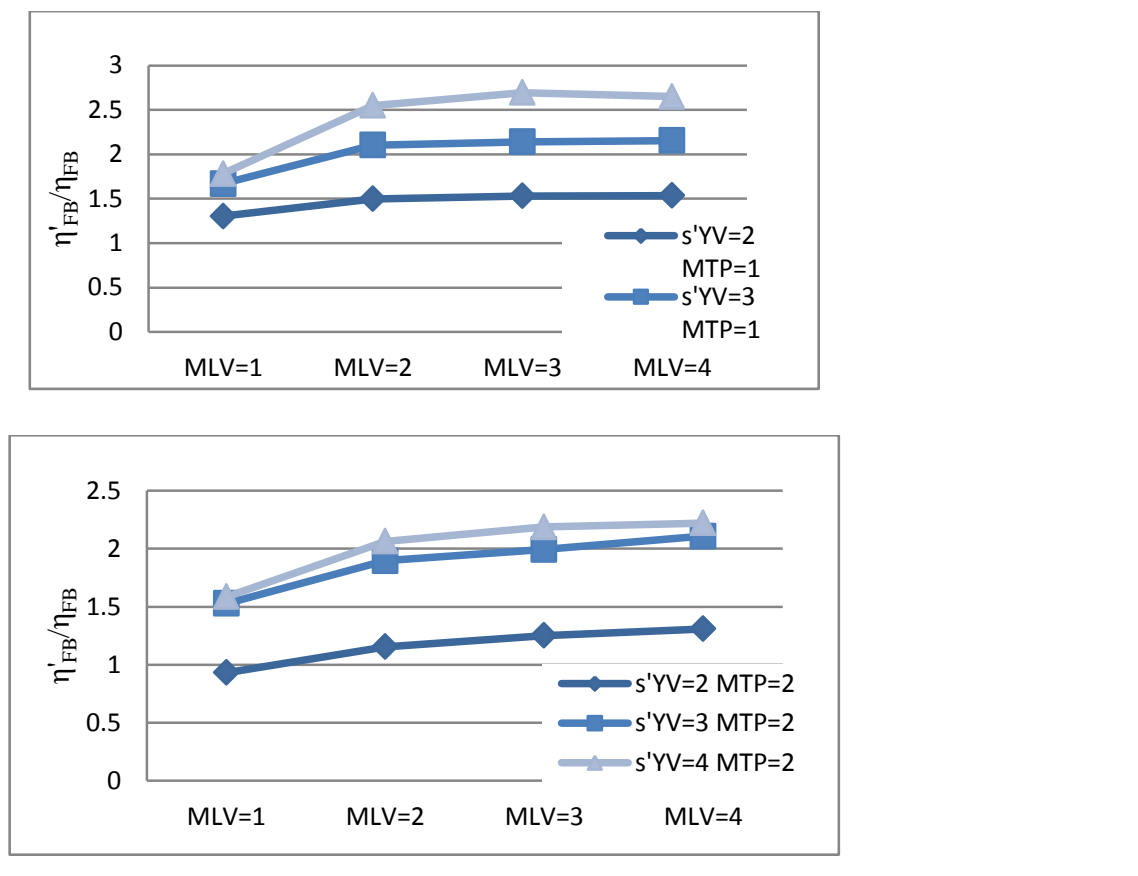

Figure 8. Comparison chart of horizontal,transport efficiency

The two chart shows, increasing the number of stories of frame bridges and the trolleys the level of transportation efficiency ratio has improved to a certain extent, because of the increased number of frame bridges, reduce the traffic jam situation. But with the increasing of the number of frame bridge layers and tranŝportyehicles in each story and each column the ratio of transportation efficiency increase sfowly, which can be analyzed, the waiting time of transport vehicles for receiving service is not the main bottleneck. In the presence of the number of yard crane and quay crane are the same, the average time that transport vehicles wait for the cranes become longer, and transportation efficiency will decline. When the number of thanspoit vehicles is not large, efficiency was not improved significantly by increasing the number of TP, because the main bottleneck of the system is not the number of $\mathrm{TP}$. On the contrary, increasing the equipment generate idle situation thus reducing the ratio of transport efficiency

\section{Conclúsions}

This pape presents a new type of layout and transport systems in automated container termina The index of storage capacity and the transportation efficiency are utilized to evaluate this system. Finally, through the case study, with the different frame bridge layers and the number of transport vehicle, comparison of transport efficiency is obtained. It proves that the scheme not only can improve the utilization rate of storage space, but also improve the efficiency of transportation equipment, so as to improve loading and unloading efficiency, and provides the reference and help for the equipment of selection in future port construction. 


\section{Acknowledgements}

This work sponsored by National Natural Science Foundation project(71101090), Shanghai Top Academic Discipline Project-management science \& engineering, Ministry of Education Projects (20133121110001), Shanghai Municipal Education Commission Project (12ZZ148, 13YZ080, 14YZ112), Ministry of Transport Research Projects (2012-329-810-180) and Shanghai Maritime University Research Project (20120102, 20120125).

\section{References}

[1] H. Lin and Q. Tang, "Loading and unloading technological schemes for new type automatic container terminals", Port and Waterway Engineering, vol. 10, (2008), pp. 30-33.

[2] M. H. Zhu, X. M. Fan, H. C. Cheng and Q. C. He, "Simulation of Automated Container Terminal Loading and Unloading Technology”, China Mechanical Engineering, vol. 20, no. 17, (2009), pp. 2072-2075.

[3] C. I. Liu, H. Jula and P. A. Ioannou, "A simulation approach for performance evaluation of proposed automated container terminals”, Intelligent Transportation Systems 2001. Proceedings 200 AEEE, (2001), pp. 563-568.

[4] F. Shi, X. Zhang and Q. Zhao, "Simulation and Analysis of a New Type of Automated Container Terminal", Machinery \& Electronics, vol. 12, (2008), pp. 016.

[5] W. Wang, Z. Q. Yao and Q. F. Bao, "Research On the Handling Technology of the Automatic Yard", Machine Design and Research, vol. 23, no. 2, (2007), pp. 84-87.

[6] Y. H. Zhao, "Research on Handling Processes of a New Type of Automation Container Terminal", Port Operation, vol. 3, (2009), pp. 22-24.

[7] L. Zhen, L. H. Lee, E. P. Chew, D. -F. Chang and Z. X. Xu, "A Comparative Study on Two Types of Automated Container Terminal Systems", IEEE Transactions on Automation Science and Engineering, vol. 9, no. 1, (2012) January, pp. 56-69.

[8] H. Y. Bae, R. Choe, T. Park and K. R. Ryu, Comparison of operations of AGVs and ALVs in an automated container terminal", J Intell Manuf., vol. 22 (2011), pp. 413426.

[9] S. Hoshino, J. Ota, A. Shinozaki, et a. "Hybrid design methodology and cost-effectiveness evaluation of AGV transportation systems", IEEE Tansactions on Automation Science and Engineering, vol. 4, no. 3, (2007), pp. 360-372.

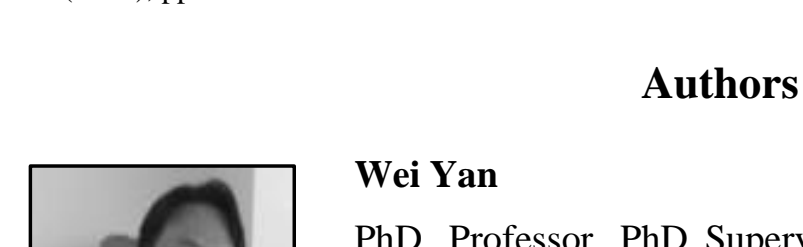

$\mathrm{PhD}$, Professor, PhD Supervisor, director of Development Planning Division. His current research interests are related to the supply chain and logistics, port and shipping, industrial engineering, spanning from conceptual design, system modeling to KE/AI-based system optimization.
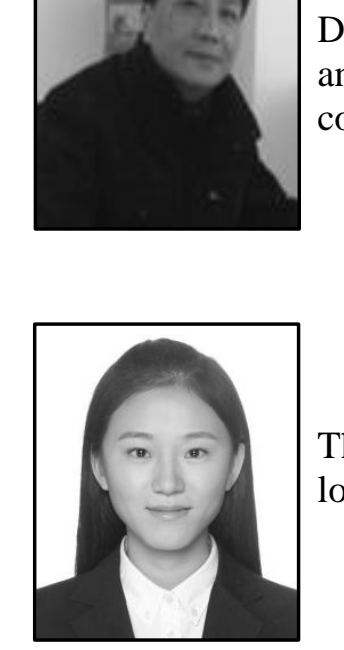

\section{Yishi Zhu}

She is currently a postgraduate student in Mechanical Design and Theory at Shanghai Maritime University. Her research interest is port logistics technology. 


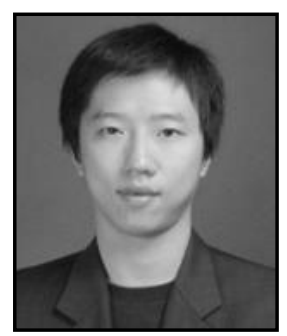

\section{Junliang He}

$\mathrm{He}$ is a lecturer at container supply chain technology Engineering Research Center in Shanghai Maritime University. Currently he is $\mathrm{PhD}$ candidate in the school of mechanical engineering of Tongji University. Main research interests include artificial intelligence, knowledge engineering.

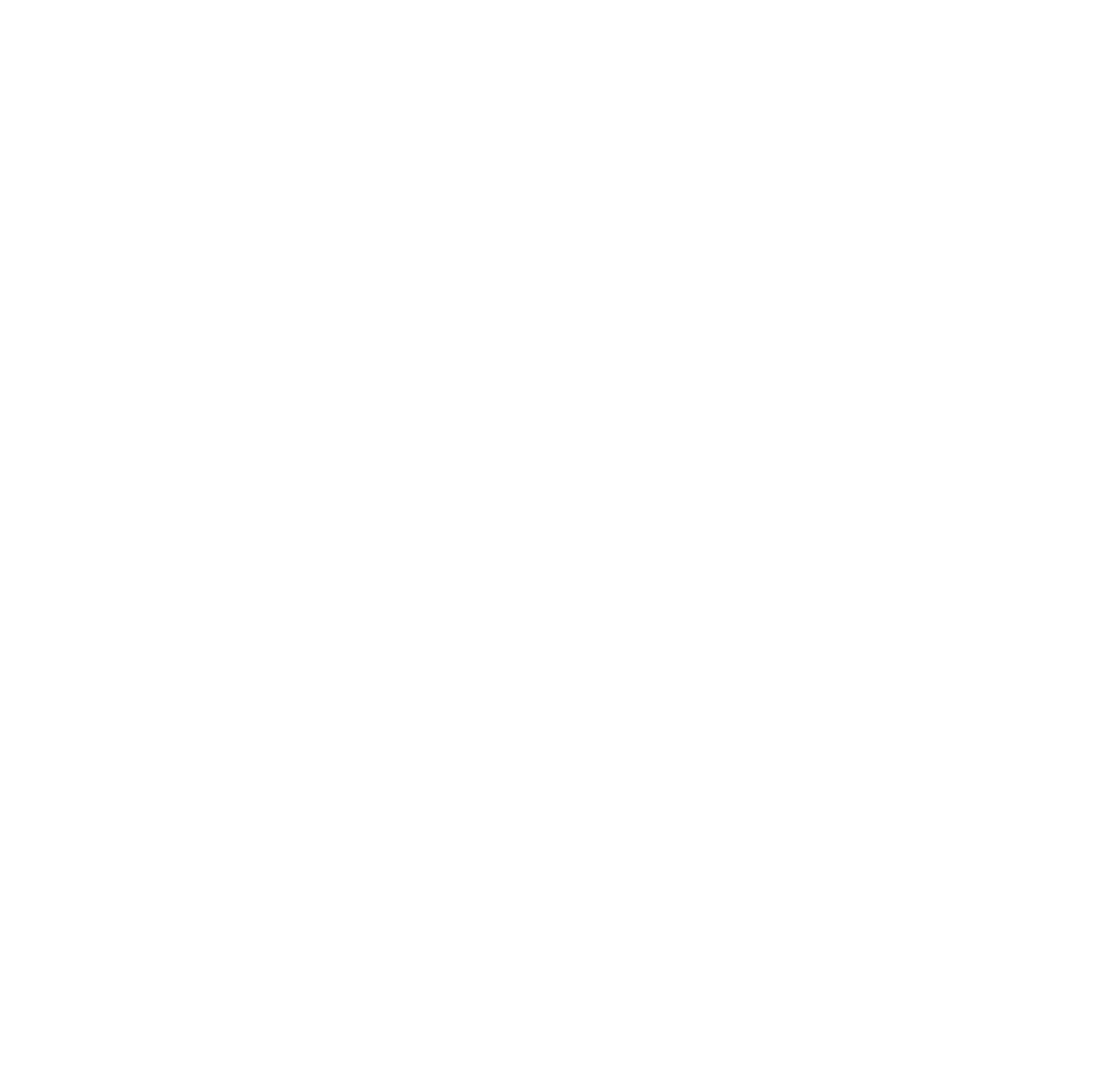

